\title{
New Pneumococcal Diagnostics---further ahead or more confused?
}

\author{
Invited Lecture Abstracts
}

\author{
ISPPD-0205 \\ New Pneumococcal Diagnostics---further ahead or more confused?
}

\section{USEFULNESS OF SEROTYPE SPECIFIC URINARY ANTIGEN DETECTION (UAD) ASSAY TO DETECT PNEUMOCOCCAL PNEUMONIA IN ADULTS}

W. Albrich ${ }^{1}$, M.W. Pride ${ }^{2}$, S.A. Madhi ${ }^{3}$ J. Callahan ${ }^{4}$, P.V. Adrian³ ${ }^{3}$ R. French ${ }^{5}$, N. van Niekerk ${ }^{6}$, S. Sebastian², V. Souza ${ }^{2}$, K.U. Jansen ${ }^{2}$, K.P. Klugman ${ }^{3}$

${ }^{1}$ Division of Infectious Diseases and Hospital Epidemiology, Cantonal Hospital St. Gallen, St. Gallen, Switzerland; ${ }^{2}$ Pfizer Vaccine Research and Early Development, Pfizer Inc., Pearl River, USA; ${ }^{3}$ Respiratory and Meningeal Pathogens Research Unit/Medical Research Council, National Institute for Communicable Diseases, Johannesburg, South Africa; ${ }^{4}$ Callahan Associates Inc, Callahan Associates Inc, La Jolla, USA; ${ }^{5}$ Biotechnology Clinical Development Statistics, Pfizer Inc., Pearl River, USA; ${ }^{6}$ Respiratory and Meningeal Pathogens Research Unit/Medical Research Council, Respiratory and Meningeal Pathogens Research Unit/Medical Research Council, Johannesburg, South Africa

Background and Aims: A urinary antigen detection assay (UAD) for 13 pneumococcal serotypes was recently reported as a useful diagnostic tool for pneumococcal pneumonia. We tested this assay in a well-defined cohort of HIV-infected South African adults with X-ray confirmed pneumonia.

Methods: A Luminex XMAP bead-based multiplex UAD detecting the PCV13 serotypes was tested retrospectively in HIV-infected South African adults with pneumonia. UAD positivity cut-offs were established in HIV-infected South Africans without respiratory disease. Pneumonia was considered pneumococcal if a composite diagnostic including any of sputum Gram stain, sputum culture, blood culture or urinary Binax was positive.

Results: The UAD identified 6/7 (85.7\%) patients with bacteremia due to a PCV13 serotype and did not misclassify any of 3 patients with bacteremia due to a non-PCV13 serotype. Even though the UAD detects only 13 serotypes, it detected more cases of presumed pneumococcal pneumonia among $\mathrm{X}$ - ray confirmed cases than the serotypeindependent urine Binax (106/235 (45.1\%) vs. 52/235 (22.1\%); $p<0.001)$. The UAD was positive in 52/71 (73.2\%) patients with a positive composite diagnostic suggesting that $73 \%$ of disease was due to PCV13 vaccine types and overall detected significantly more cases than the composite $(106 / 235$ (45.1\%) vs $71 / 235(30.2 \%) ; p<0.001)$. No correlation was detected between nasopharyngeal colonization density measured by lytA rtPCR and quantitative UAD values.

Conclusions: The UAD compared more favourably with other diagnostic tests for pneumococcal pneumonia in adults and may become a new standard for detection of pneumococcal pneumonia in adults.

Conflict of interest

ISPPD-0569

New Pneumococcal Diagnostics---further ahead or more confused?

\section{DISSECTING THE COMPLEXITY OF CARRIAGE: MOLECULAR MINING BY MICROARRAY}

J. Hinds ${ }^{1}$

${ }^{1}$ Bacterial Microarray Group, St George's University of London, London, United Kingdom

Nasopharyngeal carriage represents the natural host reservoir from which Streptococcus pneumoniae may achieve transmission to a new host or cause invasive disease. The biology of the pneumococcus has evolved for successful colonization of this complex niche, including strategies to avoid host defences and co-exist with other microbes. The dynamic microbial community of the nasopharynx is in constant flux and presents opportunities for genetic exchange, both impacting on the effectiveness of pneumococcal vaccines and antimicrobial resistance.

Diagnostic methods have revealed key insights into carriage of co-colonizing species and pneumococcal serotypes. As nasopharyngeal carriage is a precursor to invasive pneumococcal disease and has a role in herd immunity, monitoring the effect of vaccines on carriage is especially important. In the era of polysaccharide conjugate vaccines, serotyping has been a key technique to study the impact upon circulating vaccine and non-vaccine serotypes. The microarray-based molecular serotyping method has shown high specificity and sensitivity for detection of multiple pneumococcal serotypes in carriage samples. Enhanced detection of additional serotypes at low abundance may affect metrics used in assessing vaccine efficacy, such as acquisition and duration of carriage, and also case:carrier ratios. Furthermore, the ability to detect serotype variants and non-typeables along with closely related species, antimicrobial resistance determinants and pneumococcal genotype provide valuable supplementary information. This combines to help dissect the complex nasopharyngeal niche and also contextualise an individual sample within other local or global studies.

Conflict of interest 


\title{
Oral Plenary Abstracts
}

ISPPD-0516

New Pneumococcal Diagnostics---further ahead or more confused?

\section{EVALUATION OF A COMPUTERIZED PROGRAM FOR AUTOMATED STANDARDIZED CLASSIFICATION / INTERPRETATION OF CHEST X-RAY IMAGES IN THE PERCH STUDY}

\author{
W. Gong ${ }^{1}$, D.M. Eckley², M. Silaba ${ }^{3}$, J.A. Scott ${ }^{3}$, I.G. Goldberg ${ }^{2}$, K.L. O’Brien ${ }^{1}$, The PERCH Study Group ${ }^{1}$, M.D. Knoll ${ }^{1}$
}

${ }^{1}$ International Vaccine Access Center, The Johns Hopkins Bloomberg School of Public Health, Baltimore, USA; ${ }^{2}$ Laboratory of Genetics, National Institute on Aging/National Institutes of Health, Baltimore, USA; ${ }^{3}$ Epidemiology and Demography, Kilifi-KEMRI Wellcome Trust, Kilifi, Kenya

Background: Alveolar consolidation on chest radiography (CXR-AC) is used to enhance identification of bacterial pneumonia specifically in studies to estimate pneumococcal conjugate vaccine (PCV) efficacy and effectiveness. However, CXR image interpretation is labor-intensive and difficult to standardize. Computer-assisted tools may facilitate and standardize CXR assessment.

Methods: Digital CXR images from 250 children aged 28d - 59 months hospitalized with WHO-defined severe or very severe clinical pneumonia enrolled in the Pneumonia Etiology Research for Child Health (PERCH) study in Kilifi, Kenya, were classified by two trained clinicians/radiologists as WHO defined CXR-AC, other infiltrate, both, normal or uninterpretable. Discordant classifications were adjudicated by a panel of 2-3 radiologists. Image pattern recognition software (WND-CHARM) was used to train an automated classification model. Over 3000 generic image descriptors/features of CXRs were extracted including pixel statistics, high contrast features, and textures. The features were filtered and weighted using human-defined classification of 188 randomly selected training images. 62 practice images were classified based on their similarity to the training set.

Results: Binary classifiers predicted CXR-AC in the sample images with an accuracy of $78.9 \%$ (area-undercurve $=0.714$ ), indicating moderately good classification. Classifiers for other consolidation/infiltrate did not show meaningful classification power.

Conclusion: Currently the performance of computer automated CXR image classification is not optimized but it shows promise for a standardized CXR assessment in PCV impact studies. Human-defined intercostal space-specific locations of CXR-AC will be applied in model training and will likely improve the prediction performance significantly.

\section{Figure 1: ROC curve of the PEP prediction in testing CXR image set}

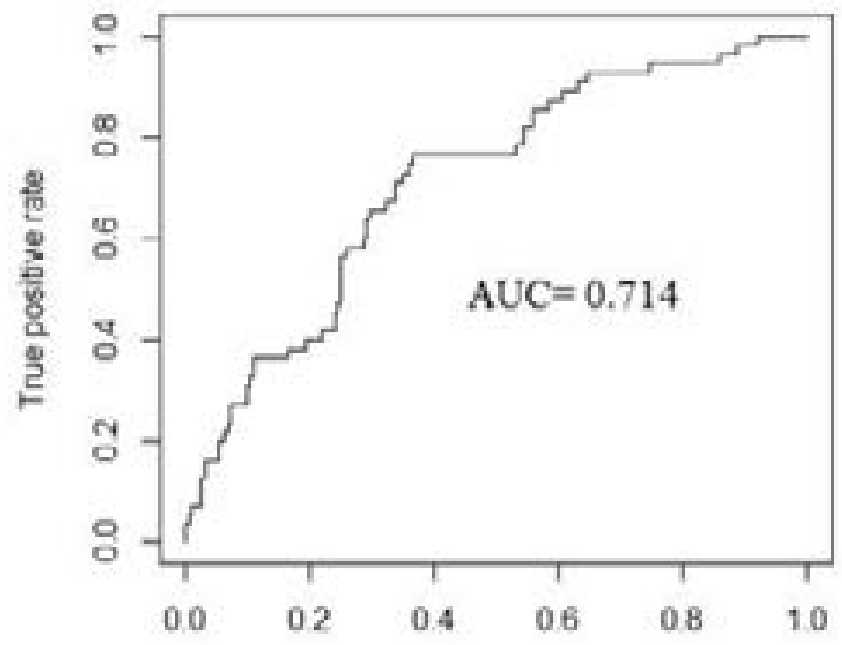

False positive rate

No conflict of interest 
ISPPD-0252

New Pneumococcal Diagnostics---further ahead or more confused?

\title{
PNEUCARRIAGE PROJECT: IDENTIFYING THE OPTIMUM PNEUMOCOCCAL SEROTYPING METHOD(S), INCLUDING DETECTION OF MULTIPLE SEROTYPE CARRIAGE
}

\author{
C. Satzke ${ }^{1}$, E.M. Dunne ${ }^{1}$, B.D. Porter ${ }^{1}$, M. Antonio ${ }^{2}$, K.L. O’Brien ${ }^{3}$, R.M. Robins-Browne ${ }^{4}$, J.A. Scott ${ }^{5}$, K.P. Klugman ${ }^{6}$, \\ E.K. Mulholland ${ }^{1}$
}

${ }^{1}$ Pneumococcal Research, Murdoch Childrens Research Institute, Parkville, Australia; ${ }^{2}$ Molecular Microbiology and Mycobacteriology, Medical Research Council Unit, Fajara, Gambia; ${ }^{3}$ International Health, Johns Hopkins Bloomberg School of Public Health, Baltimore, USA; ${ }^{4}$ Department of Microbiology and Immunology, The University of Melbourne, Parkville, Australia; ${ }^{5}$ Epidemiology and Demography, KEMRI-Wellcome Trust Collaborative Research Progamme, Kilifi, Kenya; ${ }^{6}$ Rollins School of Public Health, Hubert Department of Global Health, Atlanta, USA

Background and Aims: Nasopharyngeal carriage studies offer a practical approach for evaluating vaccines or measuring vaccine impact. A major barrier is that the current gold-standard serotyping method, the Quellung reaction, is very laborious. The PneuCarriage Project is a large, international collaboration that determined the optimum method(s) for pneumococcal serotyping, including the ability to detect multiple serotype carriage. Methods: In the first phase of the project, 15 methods groups used 20 different methods to serotype a set of 81 laboratory prepared ('spiked') samples. Five methods were selected for testing 260 nasopharyngeal swabs collected from children in six countries ('field' samples). Samples were also thoroughly characterised by traditional serotyping of $>100$ colonies.

Results: For the spiked samples, the sensitivity and positive predictive value (PPV) ranged from 0.5-99\% and 8-100\% respectively. Five methods with $\geq 70 \%$ sensitivity to detect minor serotypes, and a PPV $\geq 90 \%$, were selected for the field sample testing: microarray, real-time PCR (directly from sample or following a culture-amplification step), latex sweep and mPCR/RFLP. Preliminary analysis of field sample testing found that all methods had sensitivity $\geq 79 \%$ and PPV $\geq 90 \%$, except the real-time PCR (culture) method which had a PPV of $84 \%$. The microarray displayed the best scientific performance, with 96\% sensitivity and 92\% PPV. 105 discrepant results are being resolved by conducting blinded Quellung reactions or single-plex quantitative real-time PCR.

Conclusion: These data demonstrate the importance of rigorous evaluations of serotyping methods, and can now be used to support carriage studies evaluating vaccines or investigating vaccine impact.

No conflict of interest

ISPPD-0568

New Pneumococcal Diagnostics---further ahead or more confused?

\section{DETECTION OF STREPTOCOCCUS PNEUMONIAE BY WHOLE BLOOD LYTA PCR AND ASSOCIATION WITH PEDIATRIC PNEUMONIA}

The PERCH Study Group ${ }^{1}$

${ }^{1}$ International Vaccine Access Center, Johns Hopkins University, Baltimore, USA

Background and Aims: Detection of the autolysin-A gene (lytA) by PCR in whole blood may be useful for diagnosing pneumococcal pneumonia.

Methods: In the Pneumonia Etiology Research for Child Health (PERCH) case-control study conducted in seven African and Asian countries, whole blood was tested by PCR for lytA (WB-lytA) in children aged 28 days - 59 months hospitalized with WHO-defined severe or very severe pneumonia and in age-frequency matched controls selected randomly from the community, including $23 \%$ with upper-respiratory tract infection (URTI). The proportion with positive WB-lytA and the distribution of quantitative values among positives were compared.

Results: Among 3,334 cases and 4,118 controls, the WB-lytA positivity rate ranged between sites from $0.5-13 \%$ in cases and from 2-15\% in controls (Figure). WB-lytA positivity was significantly more common among cases than healthy controls only in Mali. WB-lytA positivity rates were lower in Asian sites compared to African sites. The distribution of quantitative values substantially overlapped between cases and controls.

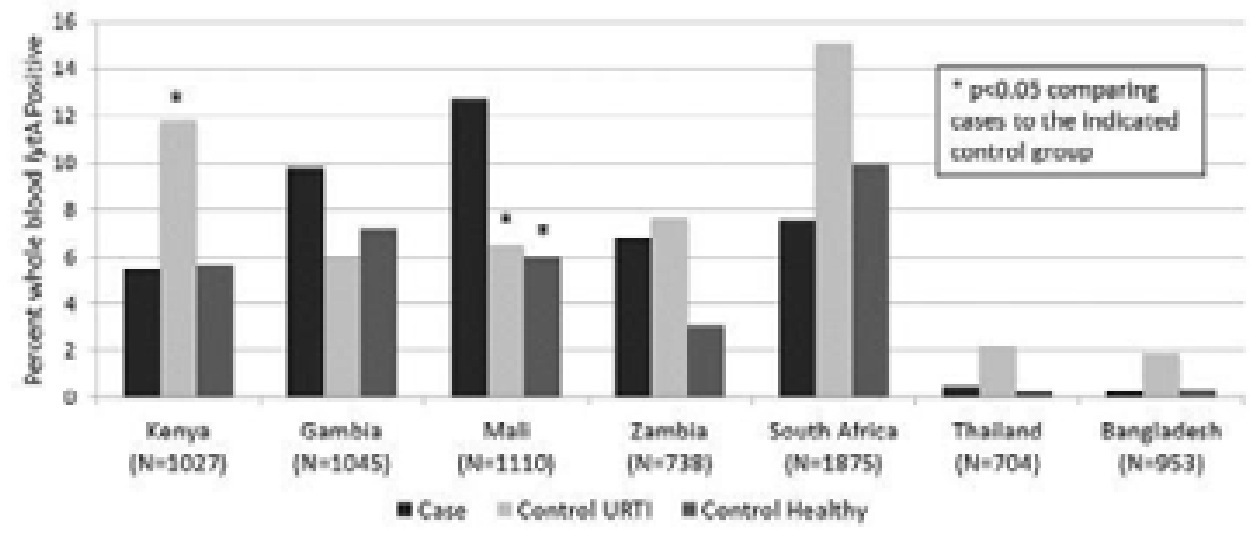


Conclusion: Results are preliminary, but the value of WB-lytA as a diagnostic tool for attributing pneumococcal etiology in children with pneumonia is questionable. Further confirmatory testing of quantitative values and exploration of potential confounding including site-specific NP density are being conducted.

No conflict of interest

\title{
Oral Poster Abstracts
}

ISPPD-0382

New Pneumococcal Diagnostics---further ahead or more confused?

\section{DIAGNOSTIC VALUE OF CHEST RADIOGRAPHY AND LABORATORY TESTING AND ATTRIBUTABLE PNEUMOCOCCAL ETIOLOGY IN COMMUNITY-ACQUIRED PNEUMONIA IN TÔNE DISTRICT, TOGO}

\author{
A. Blake ${ }^{1}$, B.M. Njanpop-Lafourcade ${ }^{1}$, J.N. Telles², A. Rajoharison², M.S. Makawa ${ }^{3}$, K. Agbenoko ${ }^{3}$, S. Tamekloe ${ }^{3}$, \\ J.E. Mueller ${ }^{4}$, H. Tall ${ }^{3}$, B.D. Gessner ${ }^{1}$, G. Paranhios-Baccalà2 ${ }^{2}$, J.C. Moïsi ${ }^{1}$ \\ ${ }^{1}$ Pneumotone, AMP, Paris, France; ${ }^{2}$ Laboratoire des Pathogènes Emergents, Fondation Mérieux, Lyon, France; ${ }^{3}$ Service d'épidémiologie, \\ Ministère de la Santé, Lomé, Togo; ${ }^{4}$ Département Biologie et Biostatistiques, Ecole des Hautes Etudes en Santé Publique, Rennes, France
}

Background and Aims: Diagnosis of non-bacteremic pneumococcal pneumonia remains difficult posing a serious limitation to vaccine impact studies. We aimed to estimate the diagnostic value of lytA real-time PCR (RTP) on whole blood for identification of pneumococcal etiology among patients hospitalized with CAP.

Methods: Patients of all ages hospitalized with clinically-defined CAP in Tône District, Togo, were enrolled over beginning May 1st 2010. Latent class analysis (LCA) pooled the results of semi-automated blood culture (SABC), whole blood lytA RTP, serum C-reactive protein, chest radiography (CXR) (based on the WHO process for lobar pneumonia/alveolar effusion), and clinical symptoms to categorize patients as likely Spn and non-likely Spn etiology. We estimated sensitivity and specificity of each test using the categories defined by LCA. Confidence intervals were obtained by bootstrap.

Results: We enrolled 1090 people over two years; 876 had results for all tests. Five LCA models provided consistent estimates for Spn prevalence and test sensitivity and specificity. Spn was the likely etiology for $39.2 \%$ to $41.1 \%$ of CAP cases across models; this proportion increased with age, from $24.4-25.0 \%$ under 5 years to $60.5-63.2 \%$ above 65 years. Whole blood lytA RTP had significantly higher sensitivity than SABC, $20.4-21.5 \%$ vs. $14.4-15.7 \%$, and both tests were highly specific, 97.1-97.9\% vs. 99.6-100\%. CXR appeared very sensitive to identify Spn etiology, 88.999.6\%, but less specific, 85.8-87.5\%.

Conclusion: CXR using WHO criteria had high sensitivity and specificity for pneumococcal pneumonia even with inclusion of all age groups and thus may be an optimal outcome for measuring vaccine impact.

No conflict of interest

ISPPD-0241

New Pneumococcal Diagnostics---further ahead or more confused?

\section{DENSITY OF UPPER RESPIRATORY TRACT COLONIZATION WITH STREPTOCOCCUS PNEUMONIAE AND ITS ROLE IN DIAGNOSING PNEUMOCOCCAL PNEUMONIA AMONG CHILDREN $<5$ YEARS}

The PERCH Study Group ${ }^{1}$

${ }^{1}$ International Vaccine Access Center, The Johns Hopkins Bloomberg School of Public Health, Baltimore, USA

Background and Aims: Previous studies suggested an association between upper airway pneumococcal colonization density and pneumococcal pneumonia.

Methods: The Pneumonia Etiology Research for Child Health (PERCH) case-control study enrolled children 28d-59m in seven African and Asian countries. Cases were hospitalized with WHO-defined severe or very severe pneumonia. Microbiologically-confirmed pneumococcal pneumonia (MCPP) cases had pneumococcus detected from a normally sterile site. Age-frequency matched controls were randomly selected from the community. Colonization density was calculated from quantitative lytA PCR on nasopharyngeal (NP)/oropharyngeal (OP) specimens.

Results: Among 48 MCPP cases at 5 sites, 46 (96\%) had pneumococcus detected in NP/OP by PCR versus $72 \%$ of non-MCPP cases and $75 \%$ of controls. Median density among MCPP cases $\left(1.17 \times 10^{7}\right.$ copies $\left./ \mathrm{ml}\right)$ exceeded that of non-MCPP cases $\left(1.0 \times 10^{5}, p<0.01\right)$ and controls $\left(1.4 \times 10^{5}, p<0.01\right)$, but distributions overlapped (Figure). This trend persisted across age groups and HIV status, but only for 3 sites (those with $>5$ MCPP cases). Colonization density $>7$ log copies/ml was more common among MCPP cases (56.3\%) than among controls (5.4\%) (site-adjusted odds ratio $(\mathrm{OR})=17.30, p<0.001$ ), with sensitivity $=56.3 \%$ and specificity $=94.6 \%$. Excluding non-colonized participants or sites without MCPP cases (Thailand, Bangladesh) produced similar results.

Conclusions: Pneumococcal colonization density in children $<5$ years was higher among MCPP cases than among other pneumonia cases and controls. Although overlapping density distributions may limit use as a diagnostic tool, densities $>7$ log copies/ml were strongly associated with MCPP status. 


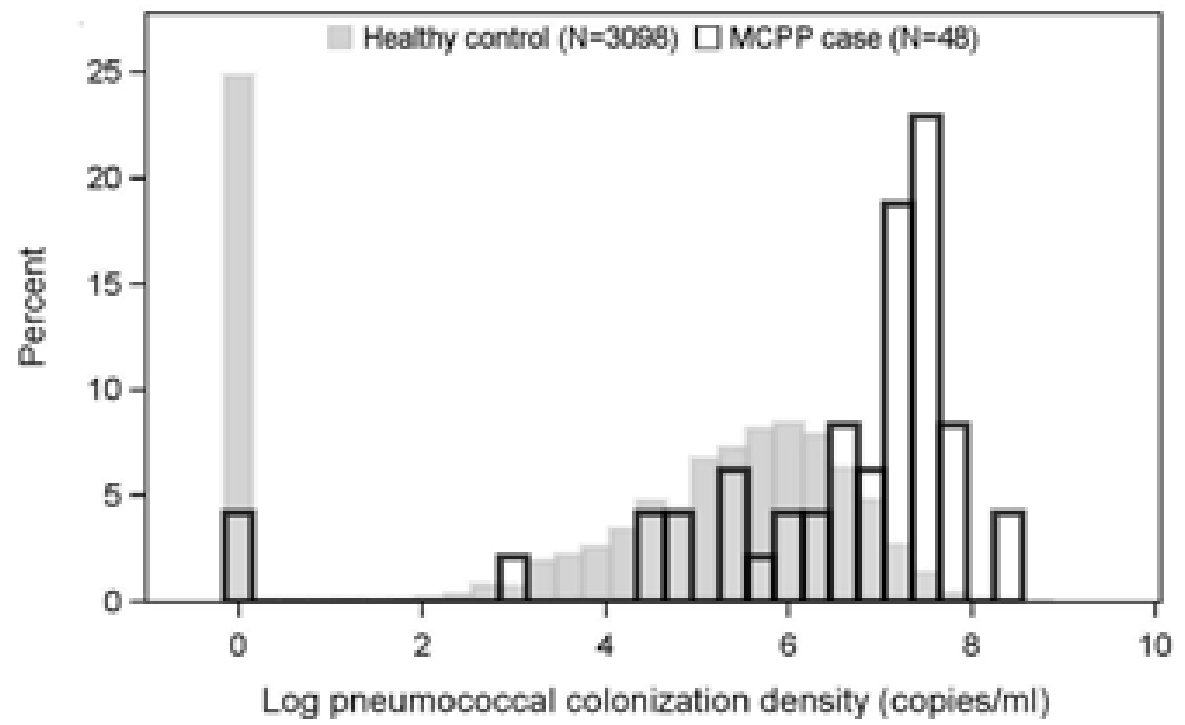

No conflict of interest

\author{
ISPPD-0086 \\ New Pneumococcal Diagnostics---further ahead or more confused?
}

\title{
PNEUMOCOCCAL DNA LOAD IN SERUM DURING BACTEREMIC PNEUMOCOCCAL DISEASE
}

\author{
A.J.H. Cremers ${ }^{1}$, F. Hagen², J.G. Ferwerda ${ }^{1}$, J.F. Meis ${ }^{2}$
}

${ }^{1}$ Dept of Pediatrics, Radboud University Medical Centre, Nijmegen, Netherlands; ${ }^{2}$ Dept of Medical Microbiology and Infectious Diseases, Canisius Wilhelmina Hospital, Nijmegen, Netherlands

Detection of pneumococcal DNA in blood could be a fast alternative for blood culture in invasive pneumococcal disease (IPD). Although its sensitivity in pneumonia may still be limited, its usability in other clinical syndromes is unknown. The value of pneumococcal DNA load detection was assessed in blood culture positive IPD cases with consideration of prior antibiotics use. Adults hospitalized with a blood culture proven pneumococcal infection between December 2008 and June 2013 were retrospectively included. Pneumococcal DNA loads in corresponding stored serum samples were determined by positive in quantitative PCR (qPCR). Data on clinical diagnosis and course of disease were extracted from medical charts. For 53 IPD cases an appropriate serum sample was retrieved. The proportion of samples qPCR was lower in uncomplicated pneumonia compared with other clinical syndromes (51.4\% vs. 92.9\%, $p=0.008)$. The pneumococcal DNA load was higher in cases of pleural empyema, meningitis and arthritis compared with uncomplicated pneumonia $(p=0.037)$ as well as in more severe disease ( $p$ values 0.006 , 0.047 and 0.004 for PSI risk class IV/V, ICU-admission and mortality respectively). Both detection of pneumococcal DNA $(p=0.3)$ and distribution of load $(p=0.6)$ did not change over the first four days of hospitalization. Detection of pneumococcal DNA in serum was more sensitive in clinical syndromes other than uncomplicated pneumonia. The pneumococcal DNA load was associated with the type of IPD and severity of disease. Since the pneumococcal DNA load seemed unaffected by antibiotic treatment during the first days of IPD, it may offer an alternative for blood culture after antibiotics use.

No conflict of interest

ISPPD-0137

New Pneumococcal Diagnostics---further ahead or more confused?

\section{QUANTITATIVE PCR TESTING OF THROAT SWAB SAMPLES AS A DIAGNOSTIC TOOL FOR PNEUMOCOCCAL PNEUMONIA IN ADULTS}

\author{
H.C. Dobinson ${ }^{1}$, T.P. Anderson ${ }^{1}$, A.M. Werno ${ }^{1}$, S. Slow², M.J. Maze ${ }^{3}$, S.T. Chambers' ${ }^{2}$, D.R. Murdoch ${ }^{2}$
}

${ }^{1}$ Microbiology, Canterbury Health Laboratories, Christchurch, New Zealand; ${ }^{2}$ Pathology, University of Otago, Christchurch, New Zealand; ${ }^{3}$ Infectious Diseases, Christchurch Hospital, Christchurch, New Zealand

Background and Aims: There is need for improved diagnostics for pneumococcal pneumonia. Preliminary data indicate that pneumococcal load in the nasopharynx may be a useful diagnostic test for pneumococcal pneumonia in adults. The aim of this study was to evaluate the utility of pneumococcal load in the oropharynx as an alternative diagnostic tool.

Method: Quantitative real-time lytA PCR was performed on archived throat swab samples from adults admitted to Christchurch Hospital (Christchurch, New Zealand) with community-acquired pneumonia as part of two studies conducted in 1999-2000 and 2012-2013. The diagnosis of pneumococcal pneumonia was based on the presence of a positive urinary antigen test and/or positive blood culture for pneumococcus.

Results: Some 69 of 248 patients (28\%) in the 1999-2000 study and 14 of 113 (12\%) in the 2012-2013 study had 
pneumococcal pneumonia. The sensitivity of the lytA PCR on throat swabs was $70 \%$ in both study groups, and specificity was $76 \%$ and $92 \%$, respectively. ROC curves for quantitative lytA PCR showed poor diagnostic accuracy (AUC $0.59(95 \% \mathrm{Cl} 0.48,0.69)$ for the combined dataset), with no obvious cut-off in pneumococcal load that would distinguish pneumococcal pneumonia from carriage. The results were unchanged when the data were stratified by prior antibiotic use, smoking, duration of symptoms and disease severity.

Conclusion: The results from this study indicate that lytA PCR testing of throat swabs has suboptimal diagnostic accuracy for detecting pneumococcal pneumonia in adults. Further work should focus on assessment of pneumococcal load in other clinical samples.

No conflict of interest

ISPPD-0224

New Pneumococcal Diagnostics---further ahead or more confused?

\section{UNI-GOLD STRETOCOCCUS PNEUMONIAE - A NEW LATERAL FLOW TEST FOR URINE SAMPLES}

\section{S. Otte ${ }^{1}$, M.S. Haugan², S. Athlin ${ }^{3}$, R. Reed ${ }^{4}$, M. Krstic ${ }^{4}$, P.L. Elverdal ${ }^{1}$, M.B. Kerrn ${ }^{1}$, I.C. Skovsted ${ }^{1}$}

${ }^{1}$ Diagnostica, Statens Serum Institut, Hillerød, Denmark; ${ }^{2}$ Department of Clinical Microbiology, Hvidovre Hospital, Hvidovre, Denmark; ${ }^{3}$ Department of Infectious Diseases, Örebro University Hospital, Örebro, Sweden; ${ }^{4}$ Research and Development, Trinity Biotech, Carlsbad CA, USA

Aim: The aim of this study was to evaluate a new lateral flow test, Uni-Gold ${ }^{\mathrm{TM}}$ Streptococcus pneumoniae, for rapid detection of all serotypes of $S$. pneumoniae in urine samples, and test for possible cross-reactions based on spiked samples. The sensitivity and the specificity of the assay was compared with BinaxNOW ${ }^{\circledR}$ S. pneumoniae, based on retrospective clinical samples.

Methods: The test uses polyclonale antibodies directed against 92 known serotypes.

Isolates of each of the 92 pneumococcal serotypes were spiked into negative urine to a final concentration at $1 \times 10^{5}$ $\mathrm{cfu} / \mathrm{mL}$ before testing with the Uni-Gold ${ }^{\mathrm{TM}}$ S. pneumoniae. Furthermore the Uni-Gold ${ }^{\mathrm{TM}}$ S. pneumoniae was tested for possible cross-reactions with 122 bacteria (65 species) spiked into negative urine to a final concentration at $1 \times 10^{6} \mathrm{cfu} / \mathrm{mL}$. In a retrospective study on clinical urine samples, 73 urine samples from patients with positive blood culture for S. pneumoniae and 225 urine samples from patients with a blood culture negative for S. pneumonia, were tested.

Results: All 92 serotypes were positive in the Uni-Gold ${ }^{\mathrm{TM}}$ S. pneumoniae, and there were no cross-reactions to the 122 spiked bacteria. For the retrospective study using blood culture as a reference, the following results were obtained:

\begin{tabular}{|c|c|c|c|}
\hline & + & - & Sensitivity \\
\hline Uni-Gold $^{\mathrm{TM}}$ S. pneumoniae & 64 & 9 & $88 \%$ \\
\hline \multirow[t]{2}{*}{ BinaxNOW ${ }^{\circledR}$ S. pneumoniae } & 59 & 14 & $81 \%$ \\
\hline & + & - & Specificity \\
\hline Uni-Gold $^{\mathrm{TM}}$ S. pneumoniae & 17 & 208 & $92 \%$ \\
\hline BinaxNOW ${ }^{\circledR}$ S. pneumoniae & 7 & 218 & \\
\hline
\end{tabular}

Conclusion: The Uni-Gold ${ }^{\mathrm{TM}}$ S. pneumoniae could be used for rapid detection of 92 S. pneumoniae serotypes in urine samples, and no expected cross-reactions were observed. Uni-Gold ${ }^{\mathrm{TM}} \mathrm{S}$. pneumoniae had a higher sensitivity than the BinaxNOW ${ }^{\circledR}$ S. pneumoniae, but a lower specificity.

Conflict of interest

ISPPD-0024

New Pneumococcal Diagnostics---further ahead or more confused?

\section{DEVELOPMENT OF A QUANTITATIVE REAL-TIME MULTIPLEX PCR ASSAY FOR 40 STREPTOCOCCUS PNEUMONIAE SEROTYPING AND ITS VALIDATION ON CLINICAL SAMPLES}

\section{G. Paranhos-Baccalà ${ }^{1}$, M. Milenkov' ${ }^{1}$, M. Messaoudi ${ }^{1}$, M.P.G. van der Linden $^{2}$, J.N. Telles ${ }^{1}$}

${ }^{1}$ Emerging Pathogens Laboratory, Fondation Mérieux, Lyon, France; ${ }^{2}$ German National Reference Center for Streptococci Department of Medical Microbiology, University Hospital RWTH, Aachen, Germany

Background and Aims: The identification of circulating Streptococcus pneumoniae strains is required to monitor the impact of the conjugate pneumococcal vaccines introduction. Conventional serotyping methods do not provide any quantitative information. Here, we describe the development a multiplex real-time PCR assay for the identification and the quantification of the 40 most prevalent S. pneumoniae serotypes and its validation on clinical samples from different countries.

Methods: A real-time multiplex assay has been developed for the detection of the 40 main S. pneumoniae serotypes. The use of external calibrators allows the quantification of each serotype. The analytical sensitivity and specificity have been evaluated on a representative panel of $S$. pneumoniae strains characterized with gold-standard methods. The assay has then been evaluated on directly on respiratory and blood samples from South Africa, Brazil, Cambodia, Mali and France. without any culture steps

Results: The S. pneumoniae real-time multiplex PCR assay showed an analytical sensitivity of $100 \mathrm{cfu} / \mathrm{ml}$ and no 
cross-reaction between each serotype or with different streptococcus species strains. The validation with the respiratory and blood samples from the different cohorts showed a specific geographical distribution of the $S$. pneumoniae serotypes. The mean serotype concentration found in the respiratory specimens was 3 logs higher than the corresponding serotype present in the blood samples.

Conclusions: By quantifying different serotypes directly from clinical samples, the assay we developed, allows to measure the different pneumococci populations in different compartment of the body. This is a valuable tool to better elucidate the relationship between carriage and invasiveness.

No conflict of interest

ISPPD-0441

New Pneumococcal Diagnostics---further ahead or more confused?

\section{SEVERITY OF PNEUMONIA IN COLORED X-RAY IMAGES}

N. Ravia Shabnam Parveen ${ }^{1}$, M. Mohamed Sathik ${ }^{2}$

${ }^{1}$ Dept of MCA, KLN College of Engineering, Madurai, India; ${ }^{2}$ Principal Dept of Computer Science, Sadakathullah Appa College, Tirunelveli, India

Background \& Aims: Colorizing the chest X-Ray image is a new approach in the field of Medical science. It is done to improve the features of the chest X-Ray instead of using the ordinary gray scale image. The Principal objective of this work is to serve the mankind, to find the depth of Pneumonia disease with the cheapest means of test like chest X-Ray. This study will enable the Doctors to find out the severity of the infection through the chest X-Ray image itself, rather than going for other expensive diagnosis tests.

Methods: The work is divided into two parts.

1. X-Ray Image is preprocessed and then undergoes colorization process.

2. Colored image is segmented and the infected features alone are extracted.

The second part is applied to both gray and colorized images.

Results: From the results, with a sample image, the colored X-Ray image detection accuracy is measured as 69.0026 percent. But whereas, for the same sample image, the gray scale X-Ray image detection showed only 56.7928 percent.

Conclusion: This article, will help the Physicians in finding the severity of the infection better than the conventional gray scale X-Ray image.

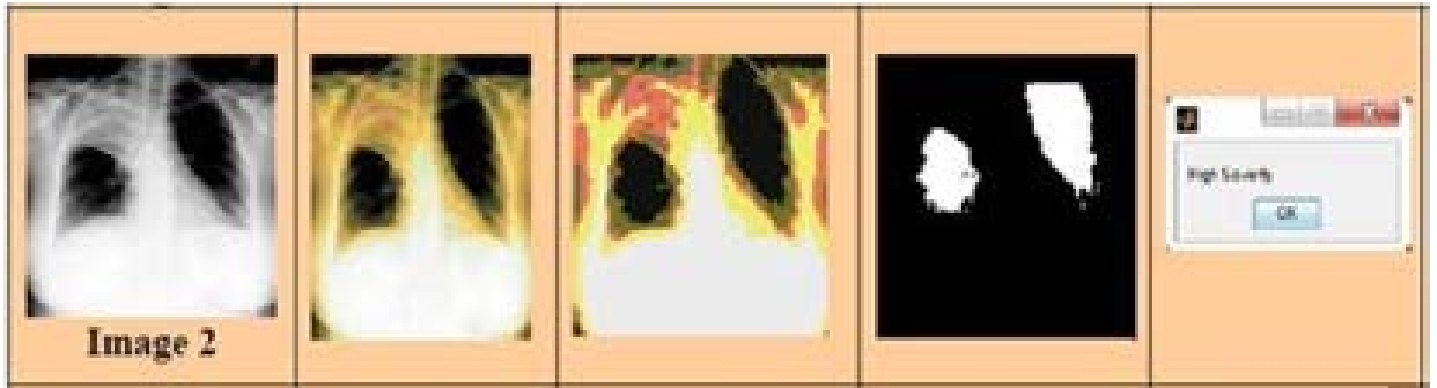

No conflict of interest

ISPPD-0362
New Pneumococcal Diagnostics---further ahead or more confused?

\section{CYCLE THRESHOLD VALUE FOR OPTIMAL IDENTIFICATION OF PNEUMOCOCCAL SEROTYPES USING MOLECULAR ASSAYS}

S. Tempia ${ }^{1}$, N. Wolter ${ }^{1}$, V. Magomani ${ }^{1}$, C. Cohen ${ }^{1}$, S. Walaza ${ }^{1}$, J. Moyes ${ }^{1}$, A. von Gottberg ${ }^{1}$

${ }^{1}$ Center for Respiratory Diseases and Meningitis, National Institute for Communicable Diseases, Johannesburg, South Africa

Background and Aims: PCR-based methods targeting specific genes of the pneumococcus, such as lytA, have resulted in improved diagnosis of pneumococcal disease. Serotyping is key for pneumococcal disease surveillance. We aimed to assess the lytA cycle threshold value (CV) for optimal serotype identification using molecular assays. Methods: We enrolled patients hospitalized with severe respiratory illness at four surveillance sites from 2009 through 2012 in South Africa. Blood was collected for pneumococcal (lytA) detection. Positive samples (C V $\leq 40)$ were serotyped using a real-time PCR assay targeting 42 common serotypes/serogroups. We compared the proportion of serotypable samples with lytA $C_{t} \mathrm{~V} \leq 30$ to the proportion of serotypable samples with individual lytA $\mathrm{C} V$ from 31 to 40 using multinomial regression.

Results: Of the 14311 patients tested $953(7 \%)$ were lytA positive and serotyping was performed on 820 (86\%; lytA C. V: range 25-40; median 36). Of these 398 (49\%) tested positive for one of the serotypes/serogroups included in the assay. The proportion of serotypable samples with $C_{t} V \leq 33$ ranged between $75 \%\left(11 / 15 ; C_{t} V 25\right)$ and $88 \%$ $(36 / 41 ; C \vee V 31)$. A progressive reduction of the proportion of serotypable samples was observed for $C_{t} V \geq 34$ : from $61 \%\left(37 / 61 ; C_{t} V 34\right)$ to $9 \%\left(4 / 45 ; C_{t} V 39\right)$. Compared to samples with $C_{t} V \leq 30$ (proportion serotypable: 77\%; 75/97), the decrease in the proportion of serotypable specimens was statistically significant for samples with individual $C_{t} V$ $\geq 35$.

Conclusions: Low bacterial load (lytA $C_{t} V \geq 35$ ) resulted in a significant loss of sensitivity of molecular serotyping assays resulting in an under-estimation of serotypable samples. 
ISPPD-0411

New Pneumococcal Diagnostics---further ahead or more confused?

\title{
SALIVA AND NASOPHARYNGEAL CARRIAGE OF STREPTOCOCCUS PNEUMONIAE IN PARENTS OF 24-MONTH-OLD CHILDREN
}

A.L. Wyllie ${ }^{1}$, L.W. Rümke ${ }^{1}$, M.L.J.N. Chu ${ }^{1}$, N.Y. Rots ${ }^{2}$, A.J. Wijmenga-Monsuur ${ }^{2}$, J.P. Bruin ${ }^{3}$, D. Bogaert ${ }^{1}$, E.A.M. Sanders ${ }^{1}$, K. Trzcinski ${ }^{1}$

${ }^{1}$ Pediatric Immunology and Infectious Diseases, Wilhelmina Children's Hospital University Medical Center Utrecht, Utrecht, Netherlands; ${ }^{2}$ Centre for Immunology of Infectious Diseases and Vaccines, National Institute for Public Health and the Environment (RIVM), Bilthoven, Netherlands; ${ }^{3}$ Diagnostic Microbiology, Regional Laboratory of Public Health, Haarlem, Netherlands

Background: Although historical records show relatively high rates of approximately $50 \%$ pneumococcal carriage in the saliva of adults (Heffron, 1939), contemporary surveillances on adult nasopharyngeal carriage report much lower rates of colonisation. Here, we compared the sensitivity of conventional culture and molecular methods for detecting pneumococcal carriage in nasopharyngeal and saliva samples in young-middle aged adults.

Methods: Trans-nasal nasopharyngeal, trans-oral nasopharyngeal and saliva samples were simultaneously collected from 299 parents of asymptomatic 24-month-old children. All samples were analysed using the standard conventional culture approach. All trans-oral and saliva samples were tested in quantitative-PCR (qPCR) targeting pneumococcal genes lytA and piaA in DNA extracted from samples culture-enriched for $S$. pneumoniae. Since we have previously shown (Trzcinski 2013, Krone ISPPD9) that molecular methods do not significantly improve detection of $S$. pneumoniae in trans-nasal samples from adults they were not tested with qPCR.

Results: By conventional culture, 29 (10\%) adults were identified as carriers, with live pneumococci isolated from 26 (9\%) trans-nasal and $4(1 \%)$ trans-oral sample cultures. Isolation of live Streptococcus pneumoniae from saliva was virtually impossible due to abundant polymicrobial culture growth. By qPCR, $89(30 \%)$ trans-oral and 84 (28\%) saliva samples were pneumococcus-positive. Altogether, 137 of 299 (46\%) adults were positive for S. pneumoniae by any method and 130 (95\%) of all 137 carriers were detected by qPCR alone.

Conclusions: Molecular detection of S. pneumoniae in culture-enriched trans-oral and saliva samples were the most sensitive methods in this study, together equally contributing to the overall $>45 \%$ detected pneumococcal carriage rate.

No conflict of interest

\section{Poster Abstracts}

ISPPD-0321

New Pneumococcal Diagnostics---further ahead or more confused?

\section{COLONIZATION DENSITY OF STREPTOCOCCUS PNEUMONIAE OF DIFFERENT SEROTYPES RELATED TO DISEASE SEVERITY IN PNEUMOCOCCAL PNEUMONIA}

\author{
H. Alpkvist ${ }^{1}$, G. Abdeldaim², J. Hedlund ${ }^{1}$, B. Herrmann ${ }^{2}$, K. Strålin ${ }^{1}$ \\ ${ }^{1}$ Department of Infectious Diseases, Karolinska University Hospital, Stockholm, Sweden; ${ }^{2}$ Department of Clinical Microbiology, Uppsala Univer- \\ sity Hospital, Uppsala, Sweden
}

Background and Aims: We aimed to study if the nasopharyngeal pneumococcal colonization density is correlated to disease severity in pneumococcal pneumonia.

Methods: In a prospective study, we enrolled patients hospitalised for radiologically confirmed community-acquired pneumonia. Nasopharyngeal aspirates were subjected to quantitative PCR for pneumococcal DNA (Spn9802) in 166 patients. Nasopharyngeal colonization densities were determined. All pneumococcal isolates were serotyped, and divided into serotype categories with high, medium, or low case fatality rate (CFR).

Results: 57 patients were considered to have pneumococcal pneumonia, as Streptococcus pneumoniae was detected by culture from blood or respiratory secretions, or by urinary antigen test. The table shows mean colonization densities according to pneumonia severity (PSI). The following proportions of severity factors were noted in cases with colonization densities of $>7.0(n=27)$ and $<7.0(n=30)$ : PSI risk class IV-V, $56 \%$ and $27 \%(p=$ $0.026)$; respiratory rate $>30,44 \%$ and $27 \%(p=0.160)$; and admission to intensive care unit, $11 \%$ and $0(p=0.061)$. Conclusion: A high nasopharyngeal colonization density was correlated to a high PSI score and could probably serve as a marker of disease severity. The correlation was seen in the serogroup category with high CFR, but not in the serogroup category with medium/low CFR. 


\begin{tabular}{|l|l|l|l|l|}
\hline \multirow{2}{*}{ Patient categories } & \multicolumn{2}{|c|}{ Mean log-10 copies/mL (standard devia- } & \\
\cline { 2 - 4 } & All cases & $\begin{array}{l}\text { PSI risk class } \\
\text { I-III }\end{array}$ & $\begin{array}{l}\text { PSI risk class } \\
\text { IV-V }\end{array}$ & \multirow{2}{*}{ p-value } \\
\hline All patients $(n=57)$ & $\begin{array}{l}6.71 \\
(1.44)\end{array}$ & $6.36(1.55)$ & $7.23(1.11)$ & 0.025 \\
\hline Serogroup high CFR $(n=22)$ & $\begin{array}{l}7.12 \\
(1.14)\end{array}$ & $6.63(1.09)$ & $7.61(1.00)$ & 0.040 \\
\hline $\begin{array}{l}\text { Serogroup medium /low CFR } \\
(n=23)\end{array}$ & $\begin{array}{l}6.82 \\
(1.05)\end{array}$ & $6.69(0.98)$ & $7.07(1.19)$ & 0.426 \\
\hline
\end{tabular}

No conflict of interest

ISPPD-0423

New Pneumococcal Diagnostics---further ahead or more confused?

\section{ECONOMIC EVALUATION OF PNEUMOCOCCAL CAPSULAR TYPING TECHNIQUES: COMPARATIVE COSTS OF MOLECULAR VS. CONVENTIONAL QUELLUNG TECHNIQUE}

T.H. Sugita ${ }^{1}$, J. Lamaro-Cardoso ${ }^{2}$, Y.M. Ternes², A. Kipnis2, M.C. Brandileone ${ }^{3}$, M.L.L.S. Guerra ${ }^{3}$, A.L. Andrade ${ }^{4}$, C.M. Toscano ${ }^{4}$

${ }^{1}$ Medical School, Federal University of Goiás, Goiania, Brazil; ${ }^{2}$ Microbiology, Institute of Tropical Pathology and Public Health, Federal University of Goiás, Goiania, Brazil; ${ }^{3}$ Microbiology, Adolfo Lutz Institute, Sao Paulo, Brazil; ${ }^{4}$ Collective Health Department, Institute of Tropical Pathology and Public Health, Federal University of Goiás, Goiania, Brazil

Background and Aims: Accurate laboratory techniques are required for pneumococcus identification and capsular serotyping (CS). Quellung reaction is the gold-standard method for CS. In Brazil, pneumococcus isolated from invasive disease surveillance are serotyped at Adolfo Lutz Laboratory Institute using Quellung reaction, referred in this study as conventional technique (CT). Recently developed CS by molecular technique (MT), using DNA extraction and multiplex real-time polymerase chain was implemented in the Applied Microbiology Laboratory of Goias Federal University. We compared MT and CT costs for pneumococcal CS.

Methods: We retrospectively collected primary cost data, for the period of January-July/2011. We used microcosting methodology considering costs of personnel, infrastructure, laboratory and office equipment and supplies. Pneumococcal identification cost was not considered, as this is a common step to both techniques. Results are presented in 2011 US dollars as total costs, stratified by categories, recurrent vs. capital costs, and costs per processed sample.

Results: (see table)

Conclusion: Significant resources are required for CS, particularly for personnel and laboratory supplies. Costeffectiveness analyses are necessary to evaluate the use of MT in selected situations.

Costs of molecular and conventional tests for pneumococcal serotyping

\begin{tabular}{|c|c|c|c|c|}
\hline \multirow[b]{2}{*}{ Components } & \multicolumn{2}{|c|}{ Molecular technique Costs } & \multicolumn{2}{|c|}{$\begin{array}{l}\text { Conventional } \\
\text { Technique Costs }\end{array}$} \\
\hline & $\begin{array}{l}\text { Total } \\
n=740\end{array}$ & Per sample & $\begin{array}{l}\text { Total } \\
n=969\end{array}$ & Per sample \\
\hline \multicolumn{5}{|l|}{ Recurrent Costs } \\
\hline Personnel & $46,508.7$ & 62.9 & $20,336.8$ & 21.0 \\
\hline $\begin{array}{l}\text { Cleaning, Electricity, Water, Communication, } \\
\text { Security, Building repairs }\end{array}$ & $7,429.7$ & 10.0 & $6,149.0$ & 6.4 \\
\hline Office supplies & 12.8 & 0.0 & 23.8 & 0.0 \\
\hline Laboratory supplies & $22,086.0$ & 27.5 & $18,771.3$ & 19.4 \\
\hline \multicolumn{5}{|l|}{ Capital Costs } \\
\hline Infrastructure (Building) & $1,478.7$ & 2.0 & 0.0 & 0.0 \\
\hline Office equipments & 36.3 & 0.1 & 26.2 & 0.0 \\
\hline Laboratory Equipments & $8,524.3$ & 11.5 & 553.5 & 0.6 \\
\hline Total & $86,076.5$ & 114.0 & $45,860.5$ & 47.3 \\
\hline
\end{tabular}

No conflict of interest 
ISPPD-0494

New Pneumococcal Diagnostics---further ahead or more confused?

\title{
MULTI-CENTER CLINICAL EVALUATION OF UNI-GOLD ${ }^{T M}$ STREPTOCOCCUS PNEUMONIAE: A NEW RAPID URINARY ANTIGEN TEST FOR PNEUMOCOCCAL CELL-WALL POLYSACHARIDES
}

S. Athlin ${ }^{1}$, H. Brander Eriksen², O. Altun ${ }^{3}$, M. Schei Haugan², V. Özenci ${ }^{3}$, K. Strålin ${ }^{4}$

${ }^{1}$ Department of Infectious Diseases, Örebro University Hospital, Örebro, Sweden; ${ }^{2}$ Department of Clinical Microbiology, Hvidovre University Hospital, Copenhagen, Denmark; ${ }^{3}$ Department of Clinical Microbiology, Karolinska University Hospital, Stockholm, Sweden; ${ }^{4}$ Department of Infectious Diseases, Karolinska University Hospital, Stockholm, Sweden

Background and Aims: A new rapid test for detecting pneumococcal cell-wall polysaccharides in urine, the UniGold $^{\mathrm{TM}}$ Streptococcus pneumoniae (Uni-Gold ${ }^{\mathrm{TM}}$ ) antigen test, was recently developed by Statens Serum Institut, Denmark, and Trinity Biotech, USA. We compared the performance of Uni-Gold ${ }^{\mathrm{TM}}$ with the BinaxNOW ${ }^{\circledR} S$. pneumoniae (BinaxNOW ${ }^{\circledR}$ ) antigen test in a multi-centre prospective clinical study. Methods: The study was performed between 20th July and 20th October 2013 in three tertiary university hospitals in Örebro (Sweden), Karolinska (Sweden) and Hvidovre (Denmark). All consecutive clinical urine samples tested with BinaxNOW ${ }^{\circledR}$ were also tested with Uni-Gold ${ }^{\mathrm{TM}}$. Positive cultures for $S$. pneumoniae on blood and airway samples were recorded.

Results: In 283 urine samples, Uni-Gold ${ }^{\top M}$ and BinaxNOW ${ }^{\circledR}$ performed similarly, both positive in 28 cases and negative in 247 cases. The results diverged in 8 cases, with no support by any culture in 7 cases (table). Thus, the correlation between the tests was very good $(\mathrm{k}=0.86)$. Based on 15 cases with pneumococcal bacteraemia, the sensitivity was $80 \%(12 / 15)$ for Uni-Gold ${ }^{\mathrm{TM}}$ and $73 \%$ (11/15) for BinaxNOW ${ }^{\circledR}$.

Conclusion: In this first evaluation study, Uni-Gold ${ }^{\mathrm{TM}}$ correlated well with BinaxNOW ${ }^{\circledR}$ on consecutive clinical urine samples, with similar performance. The present study shows that Uni-Gold ${ }^{\mathrm{TM}}$ is not inferior to BinaxNOW ${ }^{\circledR}$ in detecting $S$. pneumoniae antigen in urine samples.

\begin{tabular}{|l|l|l|l|l|l|}
\hline Positive tests & \multicolumn{5}{|c|}{ Supportive positive tests for pneumococcal aetiology } \\
\hline & Uni-Gold $^{\text {TM }}$ & BinaxNOW & & \\
& & BC & BC and/or AC & No support \\
\hline Uni-GoldTM $(n=33)^{\text {NR }}$ & 28 & 12 & 15 & 4 \\
\hline BinaxNOW $^{\circledR}(n=31)$ & 28 & NR & 11 & 14 & 3 \\
\hline
\end{tabular}

BC, blood culture; AC, airway culture: endotracheal secretion, sputum, nasopharyngeal secretion; NR, not relevant.

No conflict of interest

ISPPD-0481

New Pneumococcal Diagnostics---further ahead or more confused?

\section{RAPID IMMUNOCHROMATOGRAPHIC ASSAY FOR DETECTION OF STREPTOCOCCUS PNEUMONIAE}

\author{
E.P. Silva ${ }^{1}$, F. Nato' ${ }^{2}$, P.S. Liu ${ }^{1}$, L.F.R. Cruz ${ }^{1}$, G.C. Barazzone ${ }^{1}$, W.O. Dias ${ }^{1}$ \\ ${ }^{1}$ Center of Biotechnology, Instituto Butantan, São Paulo, Brazil; 2Plateforme 5, Institut Pasteur, Paris, France
}

Background and Aims: Traditional techniques for detecting Streptococcus pneumoniae require appropriate laboratory, time consuming and specific technical training. Immunochromatography strip tests, or namely lateral flow tests, are simple devices, using colored particles and based on capillary action, that intend to detect, in a few minutes, the presence or absence of target analytes.

Aims: The standardization of a rapid immunochromatographic assay (dipstick) for detection of S. pneumoniae. Methods: Dipsticks were developed using colored colloid microspheres or colloid gold particles, appropriately conjugated to mice monoclonal antibodies against a recombinant streptococcal pneumolysin (Ply) (Mab anti-Ply); total immunoglobulin from mice serum anti-Ply (Pab anti-Ply) or total immunoglobulin from mice serum against a whole cell pneumococcal vaccine (anti-WCPV). These antibodies were also used as capture, immobilized in a nitrocellulose membrane (MN). The sample pad, previously treated with the conjugate and pasted to the MN, was dipped in the samples, which were allowed to flow through chromatography.

Results: A dipstick assembled with colloid microspheres conjugate with anti-WCPV and captured by Mab anti-Ply showed, in $10 \mathrm{~min}$ of reaction, a limit of detection of $10^{4}$ cells $/ \mathrm{ml}$ of pneumococcus, while the made with colloid gold detected $10^{3}$ cells $/ \mathrm{ml}$. The dipstick with the conjugate colloid microspheres/ Pab anti-Ply and Mab anti-Ply as capture was able to detect at least $9.7 \mathrm{ng} / \mathrm{ml}$ of Ply in culture supernatant of S. pneumoniae, while the colloid gold dipstick detected $19.4 \mathrm{ng} / \mathrm{ml}$.

Conclusions: The results suggest the availability of the test as platform for further development of point-of-care and diagnostic applications.

Supported by FAPESP, CNPq and Butantan Foundation

No conflict of interest 
ISPPD-0409

New Pneumococcal Diagnostics---further ahead or more confused?

\title{
DETECTION OF PNEUMOCOCCAL DNA IN CHILDREN BY MULTIPLEX REAL TIME PCR, PRELIMINARY REPORT OF CLINICAL RELEVANCE IN PROSPECTIVE PIDOPS STUDY
}

\author{
A. Feroze Ganaie ${ }^{1}$, A. Vandana ${ }^{1}$, K.L. Ravikumar ${ }^{1}$ \\ ${ }^{1}$ Microbiology, Kempegowda Institute of Medical Sciences, Bangalore, India
}

Background \& Aims: Streptococcus pneumoniae is the major cause of bacteremia in children. Currently the diagnosis of pneumococcal bacteremia relies on the isolation and identification of bacteria from blood cultures. However, only a small portion of cases can be detected by conventional methods. We have embarked on a prospective study to evaluate Real Time PCR of serum for detecting Invasive pneumococcal infection in children. Methods: Invasive pneumococcal infection was suspected in 29 pediatric patients on clinical grounds, chest $X$-ray, CBC, CRP and PCT test results. Blood culture was performed in all patients. Serum from these patients were subjected to Real Time PCR assay targeting ply (Pneumolysin) and lyt-A (Autolysin) genes.

Results: Out of 29 cases 24 children were positive for CRP and PCT with increase in cell counts. Blood culture was positive in 2 of 24 cases. Real Time PCR assay was positive in 5 of 24 for both ply and lyt-A genes. All the PCR positive cases were also positive for CRP and PCT with abnormal Chest X-ray. 5 cases which were CRP and PCT negative were also culture and PCR Negative. The Real Time PCR assay was able to detect minimum of 44 gene copies/ml with $\mathrm{Ct}$ value ranging from 39.64 to 43.22 .

Conclusion: The potential of the Real Time PCR assay for clinical application was shown by its ability to detect pneumococcal infection in culture negative samples. The speed and ease of this approach will make it a useful tool for detection of pneumococci and diagnosis of disease.

No conflict of interest

ISPPD-0469

New Pneumococcal Diagnostics---further ahead or more confused?

\section{RAPID DETECTION OF STREPTOCOCCUS PNEUMONIAE IN PLEURAL FLUID SAMPLES FROM PEDIATRIC PATIENTS WITH EMPYEMA}

\author{
K. Gomez-Castellano ${ }^{1}$, J.A. Torres-Coy ${ }^{1}$, R. Ramirez ${ }^{1}$, J.H. De Waard ${ }^{1}$ \\ ${ }^{1}$ Lab. Tuberculosis, Instituto de Biomedicina, Caracas, Venezuela
}

Background and Aims: Pleural empyema is a complication of pneumonia in children. Based on culture, Streptococcus pneumoniae is the leading cause of empyema, followed by other Gram-positive bacteria. Microbiological diagnostic tests for empyema by culture frequently have false-negative results due to previous antibiotics administration. Molecular diagnosis by Polymerase Chain Reaction (PCR) and pneumococcal antigen detection by immunochromatographic testing (ICT) are reliable tools, but their diagnostic value has not been clearly established for pleural fluid samples. Pneumococcal antigen detection has only been validated for urine and cerebrospinal fluid samples. The purpose of the study was to assess the diagnostic value of pneumococcal antigen detection by ICT in pleural fluid samples of children with empyema.

Methods: Pleural fluid specimens were collected from 18 children under 5 years old with pleural empyema.

Standard culture, pneumococcal antigen detection by immunochromatographic testing (BinaxNOW@Streptococcus pneumoniae), and PCR (for lytA and cPsA) were performed on these specimens.

Results: Of the 18 cases of pleural empyema, only 1 (5.5\%) was microbiologically documented by culture. An additional 6 (33.3\%) cases, were diagnosed by PCR including the culture positive case. The ICT confirmed these 6 cases and detected pneumococcal antigens in 9 additional specimens.

Conclusion: The ICT provided a diagnosis of pneumococcal disease in 15/18 patients with empyema. Pneumococcal antigen detection in pleural fluid specimens from children provides a rapid, simple, and sensitive method of diagnosis of pneumococcal empyema, which can be confirmed by PCR when culture results are negative. Studies with larger numbers of samples are needed to validate our results

No conflict of interest 
ISPPD-0570

New Pneumococcal Diagnostics---further ahead or more confused?

\section{PNEUMOLYSIN AND AUTOLYSIN PRODUCING STREPTOCOCCUS PNEUMONIAE IN INVASIVE INFECTIONS}

R. Kanungo ${ }^{1}$, S. Anandhalakshmi ${ }^{1}$, C. Sheeladevi ${ }^{1}$, K. Prashanth ${ }^{2}$

${ }^{1}$ Clinical Microbiology, Pondicherry Institute of Medical Sciences, Pondicherry, India; ${ }^{2}$ Dept. of Biotechnology, Pondicherry University, Pondicherry, India

Pneumolysin has been attributed as a virulence factor in the pathogenesis of invasive pneumococcal infection as shown in several animal studies. Production of pneumolysin in clinical isolates have also been documented. Aim of this study was to detect pneumolysin, autolysin and PbpA in clinical isolates of Streptococcus pneumonia from invasive pneumococcal disease, and correlate with clinical outcome. S. pneumoniae isolated from blood of four patients with invasive infctions were identified by standard criteria. Molecular characterization by PCR was done to detect the genes for pneumolysin $(p / y)$, autolysin (/ytA ) and penicillin binding protein A $(P b p A)$ using following primers:

PbpA - 789bp

F - 5'CCGTATCCTGGGAGCTTTCTT 3'

R - 5'TCGCGGTTTGTTTCTACTGC 3'

LytA - 319bp

F - 5' CAACCGTACAGAATGAAGCGG 3'

R - 5' TTATTCGTGCAATACTCGTGCG 3'

Ply - 348bp

F - 5'ATTTCTGTAACAGCTACCAACGA 3'

R - 5'GAATTCCCTGTCTTTTCAAAGTC 3'

Results: Table shows details of the patients, outcome and genes detected.

\begin{tabular}{|l|l|l|l|l|l|l|}
\hline & Diagnosis & $\begin{array}{l}\text { Age } \\
\text { months }\end{array}$ & Ply gene & lytA gene & PbpA gene & Outcome \\
\hline IBT1721 & Pneumonia/Bacteraemia & 35 & + & + & + & Recovered \\
\hline IBT1960 & Fever with seizures & 72 & + & + & + & Died \\
\hline IBT1975 & ARDS & 47 & + & + & + & Died \\
\hline IBT 1793 & Pneumonia & 12 & + & + & + & Recovered \\
\hline
\end{tabular}

None of the isolates were resistant to Betalactam antibiotics tested despite the presence of $\mathrm{PbpA}$ gene. The three adult patients from whom $S$. pneumoniae with pneumolysin gene, had turbulent clinical course. Despite specific antibiotic therapy two of the patients expired within 72 hours of hospital admission. The child who had infection with S. pneumoniae without pneumolysin gene recovered completely following treatment with cefotaxime. Conclusion: Molecular characterization of four clinical isolates suggests that pneumolysin and other virulence genes can be used as prognostic markers in invasive pneumococcal disease. Proof of concept studies are needed with larger number of cliical isolates to substantiate our findings.

No conflict of interest

ISPPD-0539

New Pneumococcal Diagnostics---further ahead or more confused?

\section{EVALUATION OF BINAXNOW, STREPTOCOCCUS PNEUMONIAE URINARY ANTIGEN TEST IN CHILDREN WHO HAVE BEEN VACCINATED WITH PNEUMOCOCCAL CONJUGATE VACCINE}

Z. Kurugol ${ }^{1}$, F. Koc ${ }^{1}$, A.S.L.I. Aslan ${ }^{1}$, K. Guldane ${ }^{1}$, S. Aksit ${ }^{1}$

${ }^{1}$ Pediatrics, Ege University, Izmir, Turkey

Background and Aims: A commercial rapid urinary pneumococcal antigen test (BinaxNOW, Porland, USA) have been used in diagnosis of pneumococcal infection in children. However, urine samples can remain positive for weeks after a pneumococcal infection, and the test will also be affected if the child has been vaccinated with a pneumococcal conjugate vaccine shortly before the test. We aimed to evalute the influence of 7 valent pneumococcal conjugate vaccine on the results of the antigen detection test in children.

Method: This study was performed in Well-Child Care Clinic at Ege University Medical School between September 2008 and November 2009 in Izmir, Turkey. A total of 70 non-nasopharyngeal pneumococcal carriage healthy children who aged 15 to 60 months were enrolled in the study. Urine samples were collected from children after the vaccination with 7 valent pneumococcal conjuge vaccine ( 2 and 7 day) and a rapid urinary test (BinaxNOW) has been used to determine Streptococcus pneumoniae urinary antigen.

Results: None of the children was observed S. pneumoniae urinary antigen with BinaxNOW test after the vaccination with 7 valent pneumococcal conjuge vaccine

Conclusion: This study shows that vaccination with 7 valent pneumococcal conjugate vaccine is not influence on the results of the antigen detection test in children with BinaxNOW tests. 
ISPPD-0264

New Pneumococcal Diagnostics---further ahead or more confused?

\title{
APPLICATION OF A TARGET ENRICHMENT BASED NEXT GENERATION SEQUENCING PROTOCOL FOR IDENTIFICATION AND SEQUENCE BASED PREDICTION OF PNEUMOCOCCAL SEROTYPES
}

\author{
V. Liyanapathirana ${ }^{1}$, I. Ang ${ }^{1}$, D. Tsang ${ }^{2}$, K. Fung ${ }^{3}$, T.K. Ng${ }^{4}$, H. Zhou ${ }^{1}$, M. Ip ${ }^{1}$ \\ ${ }^{1}$ Department of Microbiology, The Chinese University of Hong Kong, Shatin, Hong Kong China; ${ }^{2}$ Queen Elizabeth Hospital, Hong Kong China; \\ ${ }^{3}$ United Christian Hospital, Hong Kong China; ${ }^{4}$ Princess Margaret Hospital, Hong Kong China
}

Background and Aims: Pneumococcal serotyping is instrumental in both diagnoses and surveillance of pneumococcal infection. While whole genome sequencing (WGS) is an attractive option, the associated cost and complex bioinformatics pipelines prevent the application of WGS in diagnostic microbiology. Target enrichment based next generation sequencing is a cost effective alternative to WGS in diagnostic microbiology applications. The aim of this study was to develop such a method capable of serotyping the common pneumococcal serotypes. Methods: Previously described primers were used in a single multiplex PCR reaction capable of identifying 40 common pneumococcal serotypes to serotype or related group levels. The resultant products which differed from each other in lengths too narrow for gel differentiation were identified based on sequences. A modified step out PCR was used to introduce unique tags enabling the pooling of multiple samples per run. Library preparation by TruSeq library preparation kit was followed by sequencing on Miseq (Illumina) platform.

Results: Correct identification of Streptococcus pneumoniae and prediction of serotypes was achieved for all isolates with $>500$ reads mapped against serotype sequences. A proportion based criteria enabled the identification of two isolates present in the same sample indicating the possibility detecting co-colonizing serotypes.

Conclusion: We propose that this method as an affordable and adaptable alternative to WGS at a diagnostic microbiology laboratory, for pneumococcal identification and serotyping.

Acknowledgements: Mr Jerris Chang and Dr. Wei-Chi Wang, Health Genetech, Taiwan for technical assistance. Illumina (Hong Kong) for partial support.

Reference: Kong F, et al: JClinMicrobiol 2006, 44:1887-1891.

No conflict of interest

ISPPD-0155

New Pneumococcal Diagnostics---further ahead or more confused?

\section{HIGH THROUGHPUT PNEUMOCOCCAL SEROTYPING}

\author{
J. Martinez ${ }^{1}$, M.H. Hickey ${ }^{1}$, J.C. Bouloutte Aristide ${ }^{1}$, U. Shaligram ${ }^{2}$ \\ ${ }^{1}$ Laboratory, $\mathrm{f}(\mathrm{x})$ Immune Co., McDonough, USA; ${ }^{2}$ Serum Institute of India, Pune, India
}

Background and Aims: There are more than 93 different serotypes of Streptococcus pneumoniae based on capsular polysaccharides (PS) identified by the 'gold standard' Quellung reaction. This technique has relatively low sensitivity, is subjective, and time consuming. We have developed a multiplexed assay capable of detection of pneumococcal PS derived from NP swab transport media, and culture isolates. Our current assay is capable of detecting serotypes $1,2,3,4,5,6 \mathrm{~B}, 7 \mathrm{~F}, 8,9 \mathrm{~N}, 9 \mathrm{~V}, 10 \mathrm{~A}, 11 \mathrm{~A}, 12 \mathrm{~F}, 14,15 \mathrm{~B}, 17 \mathrm{~F}$ 18C, 19F, 19A, 20, 22F, 23F, 33F, and CWPS.

Method: The assay is a competitive inhibition assay. Assay buffer treated samples are added to wells containing our PS conjugated bead set. MoAb reagent is added to each well to be tested. Free PS present in the sample competes with the bead bound PS for reaction with the specific MoAb resulting is loss of mean fluorescence index (MFI) signal. The loss in MFI is used to determine the serotype(s) present in the sample.

Results: This multiplexed bead assay correctly identified $90 \%$ of serotypes tested $(n=276)$. Our assay uses MoAb for 23 serotypes which have minimal cross reactivity with serogroup PS. Studies utilizing PS spiked urine demonstrate the assay's capability to detect PS in very concentrations (less than $10^{-9}$ ).

Conclusions: This assay is a quick, accurate method for performing pneumococcal serotyping. The assay is capable of high sample throughput with minimal sample manipulation, low subjectivity, good specificity, and can detect PS in urine samples.

No conflict of interest 
ISPPD-0482

New Pneumococcal Diagnostics---further ahead or more confused?

\title{
THE IMPORTANCE OF MOLECULAR DIAGNOSIS IN SEVERE SEPSIS DETERMINED BY STREPTOCOCCUS PNEUMONIAE IN ICU
}

M.M. Merisescu' ${ }^{1}$, A. Streinu-Cercel ${ }^{2}$, G. Jugulete ${ }^{1}$, D. Florea ${ }^{3}$, E. Osman ${ }^{1}$, S. Schiopu ${ }^{1}$, M. Luminos $^{1}$

${ }^{1}$ Pediatric Intensive Care, National Institute for Infectious Diseases “Prof. Dr. Matei Bals," Bucharest, Romania; ${ }^{2}$ National Institute for Infectious Diseases "Prof. Dr. Matei Bals," Bucharest, Romania; ${ }^{3}$ Genetic Laboratory, National Institute for Infectious Diseases "Prof. Dr. Matei Bals," Bucharest, Romania

Background and Aims: Infections caused by Streptococcus pneumoniae are an important cause of infant mortality worldwide. It takes many clinical forms from simple respiratory infections to severe form of sepsis, fatal in many cases. Plex-ID is the newest method in etiologic diagnosis of bacterial infections.

Methods: We conducted a 30 months study from January 2011 to July 2013 in the pediatric ICU of The National Institute of Infectious Diseases, Bucharest. The children were admitted for severe forms of pneumococcal sepsis. Positive diagnosis of sepsis was established with classic methods like clinical and laboratory criteria (hemocultures, CSF cultures), as well as Plex-id detection. The patients were split in two groups based on the above criterias.

Results: In the 30 months of study, 26 children were addmited in the hospital for pneumococcal severe infections. In the first study group, were included 15 patients with severe sepsis. The diagnosis was establised in these cases with classic methods. The mortality was 33\% (5 of 15 cases). The second study group contains 11 patients in whom we obtained 10 positive results $(90.9 \%)$ by molecular methods that were correlated with conventional methods of diagnosis.

Conclusions: The S. pneumoniae was found in the first 6 hours from admission and this lead to a decrease in mortality from $33 \%$ to $9 \%$. The major advantage of this new method is the possibility to establish the etiological diagnosis in the early hours of patient admission.

No conflict of interest

ISPPD-0326

New Pneumococcal Diagnostics---further ahead or more confused?

\section{VALUE OF THE BINAXNOW ${ }^{\circledR}$ CARD TEST IN THE QUICK IDENTIFICATION OF STREPTOCOCCUS PNEUMONIAE, IN PAEDIATRIC PATIENTS AT HARARE CENTRAL HOSPITAL, ZIMBABWE}

\author{
S. Mtapuri-Zinyowera ${ }^{1}$, S.T. Ngwende ${ }^{1}$, J. Macharaga², S. Chimhuya ${ }^{3}$, L. de Gouveia ${ }^{4}$, V. Jacha ${ }^{5}$, K.J. Nathoo ${ }^{3}$
}

${ }^{1}$ Laboratory, National Microbiology Reference Laboratory, Harare, Zimbabwe; ${ }^{2}$ Laboratory, Harare Central Hospital, Harare, Zimbabwe 3Pediatric Ward, University of Zimbabwe, Harare, Zimbabwe; ${ }^{4}$ Laboratory, National Institute of Communicable Diseases, Johannesburg, South Africa; ${ }^{5}$ Data, Harare Central Hospital, Harare, Zimbabwe

Background and Aims: The most common pathogen causing paediatric bacterial meningitis (BM), identified at Harare Central Hospital sentinel site in Zimbabwe is Streptococcus pneumoniae sero group 14. Culture-based identification of pathogens causing BM has poor specificity, therefore it is important to rapidly identify this pathogen by means of a rapid test. BinaxNOW ${ }^{\circledR}$ S. pneumoniae (Alere) is a rapid in vitro immunochromatographic assay for the qualitative detection of $S$. pneumoniae antigen.

Methods: A total of 689 cerebral spinal fluid samples were tested using the BinaxNOW ${ }^{\circledR}$ antigen card rapid test kit, culture on $5 \%$ blood and chocolate agar and measurement of glucose were done. The sensitivity and specificity of this rapid test kit was done including correlations with glucose levels.

Results: The sensitivity was $100 \%$ and the specificity was $47 \%$ using available culture as the gold standard. Nine samples were BinaxNOW ${ }^{\circledR}$ positive but culture negative. Seven out of the $8(88 \%)$ culture positive for $\mathbf{S}$. pneumoniae and 13 out of the 18 BinaxNOW $^{\circledR}$ now positive had a glucose level $<40 \mathrm{mg} / \mathrm{dl}$ (Normal $-40-85 \mathrm{mg} / \mathrm{dl}$ ). Conclusion: BinaxNOW ${ }^{\circledR}$ S. pneumoniae antigen card provides a useful adjunct to culture based diagnosis and allows for the rapid detection of pneumococcal meningitis leading to improved patient outcome in a short period of time. There is need to compare with more sensitive methods such as molecular techniques in calculation of the specificity. Low glucose levels are another indicator of the presence of S. pneumoniae.

No conflict of interest

ISPPD-0228

New Pneumococcal Diagnostics---further ahead or more confused?

\section{DOES PNEUMOCOCCUS VACCINATION HAVE ANY AFFECT ON THE RESULT FOR THE UNI-GOLD ${ }^{\text {TM }}$ STREPTOCOCCUS PNEUMONIAE LATERAL FLOW TEST?}

S. Otte ${ }^{1}$, P.L. Elverdal ${ }^{1}$, I.C. Skovsted ${ }^{1}$, M.B. Kerrn ${ }^{1}$

${ }^{1}$ Diagnostica, Statens Serum Institut, Hillerød, Denmark

Background and Aims: The aim was to evaluate whether vaccination with the 13-valent pneumococcal conjugate vaccine (Wyeth Pharmaceuticals. Inc. USA) or the 23-valent pneumococcal polysaccharide vaccine (Merck \& Co. Inc. USA) had any influence on the test result of the Uni-Gold ${ }^{\mathrm{TM}}$ Streptococcus pneumoniae test.

Methods: Fourteen healthy adults were vaccinated with the 13 -valent vaccine and 14 healthy adults vaccinated with the 23-valent vaccine, 10 urine samples were collected from each person. The first urine sample was collected 
before vaccination, and the following nine urine samples were collected each morning the following nine days. The urine samples were tested in the Uni-Gold ${ }^{\mathrm{TM}}$ S. pneumoniae test.

Results: All 28 test person's urine samples were negative before vaccination. For 27 of the test persons, the vaccination had no influence on the test result of the Uni-Gold ${ }^{\mathrm{TM}} \mathrm{S}$. pneumoniae test. One test person vaccinated with the 13-valent pneumococcal conjugate vaccine had positive urine sample the day after vaccination, but the third urine sample (collected on day two after vaccination) was negative.

Conclusion: The 13-valent pneumococcal conjugate vaccine and the 23-valent pneumococcal polysaccharide vaccine have no influence on the test result of the Uni-Gold ${ }^{\mathrm{TM}}$ S. pneumoniae test 36 hours after vaccination. Acknowledgements Thank you to Jens Juhl Otte, Md, GP, for helping collecting the clinical samples.

Conflict of interest

ISPPD-0388

New Pneumococcal Diagnostics---further ahead or more confused?

\title{
IMMULEXTM STREPTOCOCCUS PNEUMONIAE - A NEW LATEX AGGLUTINATION TEST FOR RAPID DETECTION OF STREPTOCOCCUS PNEUMONIAE IN BLOOD CULTIVATIONS AND CULTIVATIONS
}

S. Otte ${ }^{1}$, M.S. Haugan², M.B. Kerrn ${ }^{1}$, P.L. Elverdal ${ }^{1}$

${ }^{1}$ Diagnostica, Statens Serum Institut, Hillerød, Denmark; ${ }^{2}$ Department of Clinical Microbiology, Hvidovre Hospital, Hvidovre, Denmark

Background and Aims: The aim of this study was to evaluate a new latex agglutination test, ImmuLex ${ }^{\mathrm{TM}} S$. pneumoniae, for rapid detection of all serotypes of Streptococcus pneumoniae in blood cultivation and directly from plate.

Methods: The test uses polyclonale antibodies directed against 92 known serotypes. 155 S. pneumoniae positive blood cultivations were tested along with 10 negative blood cultivations in ImmuLex ${ }^{\mathrm{TM}}$ S. pneumoniae. The latex assay was compared to Remel ${ }^{\mathrm{TM}}$ Wellcogen ${ }^{\mathrm{TM}}$ Streptococcus pneumoniae latex kit.

Results: For the prospective study using blood culture as a reference, the following results were obtained:

\begin{tabular}{|l|l|l|l|}
\hline & + & - & Sensitivity \\
\hline ImmuLex $^{\mathrm{TM}}$ S. pneumoniae & 155 & 0 & $100 \%$ \\
\hline Wellcogen S. pneumoniae & 53 & 1 & $98 \%$ \\
\hline & + & - & Specificity \\
\hline ImmuLex $^{\mathrm{TM}}$ S. pneumoniae & 0 & 10 & $100 \%$ \\
\hline Wellcogen S. pneumoniae & 0 & 10 & $100 \%$ \\
\hline
\end{tabular}

Additionally, samples used in the Wellcogen kit, must be centrifuged for 5-10 minutes and a positive agglutination should be seen within 3 minutes. The slide time for ImmuLex ${ }^{\mathrm{TM}}$ is $5-10 \mathrm{sec}$ and there is no sample preparation. Additional data about cross-reactions and comparison with other kits will be presented together with data from evaluation of ImmmuLex ${ }^{T M}$ tested on pure culture.

Conclusion: It can be concluded that the new ImmuLex ${ }^{\mathrm{TM}}$ S. pneumoniae can be used as a rapid detection of $S$. pneumoniae infections, and that ImmuLex ${ }^{\mathrm{TM}}$ S. pneumoniae have a higher sensitivity than Wellcogen. The specificity was the same. Furthermore, ImmuLex ${ }^{\mathrm{TM}}$ S. pneumoniae gave a much faster positive result, and ImmuLex ${ }^{\mathrm{TM}} S$. pneumoniae had no sample preparation.

Conflict of interest

ISPPD-0226

New Pneumococcal Diagnostics---further ahead or more confused?

\section{RAPID DETECTION OF STREPTOCOCCUS PNEUMONIAE IN SPIKED SHEEP BLOOD CULTURES}

\author{
S. Otte ${ }^{1}$, I.C. Skovsted ${ }^{1}$, M.B. Kerrn' ${ }^{1}$, P.L. Elverdal ${ }^{1}$
}

${ }^{1}$ Diagnostica, Statens Serum Institut, Hillerød, Denmark

Background and Aims: The aim with this study was to develop and evaluate a latex agglutination assay (ImmuLex ${ }^{\mathrm{TM}}$ S. pneumoniae) for rapid detection of all serotypes of Streptococcus pneumoniae in blood cultures. The assay was also compared with Slidex ${ }^{\circledR}$ (Biomérieux).

Methods: Ninety-two serotypes of S. pneumoniae were cultured in a concentration of $10 \mathrm{cfu} / \mathrm{mL}$ in sheep blood in BacTec blood culture bottles, one for each serotype. Furthermore, there were tested for cross-reactions with 10 Enterococcus faecalis, 10 E. faecium, 16 Klebsiella oxytoca/pneumoniae, 10 Streptococcus group A, B, C, D and G, 5 Staphylococcus aureus, 5 Salmonella and 5 Escherichia coli. All culturing bottles were tested in ImmuLex ${ }^{\mathrm{TM}} S$. pneumoniae.

Results: 92 serotypes were positive within 10 seconds. Unfortunately two of the Klebsiella blood cultures showed a positive reaction with the latex product, when the samples were not centrifuged. After centrifugation the crossreactions were eliminated. ImmuLex ${ }^{\mathrm{TM}}$ S. pneumoniae catches 92 serotypes compared to the 82 serotypes Slidex ${ }^{\circledR}$ (Biomérieux) detected. Additionally, samples used in the Slidex ${ }^{\circledR}$ kit, must be centrifuged for 10 minutes and a positive agglutination should be seen within 2 minutes. The slide time for ImmuLex ${ }^{\mathrm{TM}}$ is 5-10 seconds and there is no sample preparation. 
Additional data about cross-reactions and comparison with other kits will be presented.

Conclusion: It can be concluded that the new ImmuLex ${ }^{\mathrm{TM}} \mathrm{S}$. pneumoniae can be used as a rapid detection of S. pneumoniae infections, and that ImmuLex ${ }^{\top M}$ S. pneumoniae could detect more serotypes than the Slidex ${ }^{\circledR}$. Furthermore, it gave a much faster positive result.

Conflict of interest

ISPPD-0525

New Pneumococcal Diagnostics---further ahead or more confused?

\section{ASSOCIATION OF STREPTOCOCCUS PNEUMONIAE, DETECTED BY CULTURE OR PCR OF INDUCED SPUTUM AND NASOPHARYNGEAL SWABS, WITH PEDIATRIC PNEUMONIA}

The PERCH Study Group ${ }^{1}$

${ }^{1}$ International Vaccine Access Center, The Johns Hopkins Bloomberg School of Public Health, Baltimore, USA

Background and Aims: We investigated whether detection of Streptococcus pneumoniae (Spn) in induced sputum (IS) and nasopharyngeal swabs (NPS) was associated with pneumonia.

Methods: PERCH is a case-control study in seven African and Asian countries among hospitalized children aged $28 \mathrm{~d}$

- 59 months with WHO-defined severe or very severe pneumonia. Age-frequency matched controls were selected randomly from the community. NPS collected from cases and controls and IS (meeting quality criteria) collected from cases were analyzed for presence of Spn by culture and lytA PCR. CXRs in cases were classified according to WHO criteria for alveolar consolidation (CXR-AC). A subset of cases unlikely to have pneumonia was defined based on absence of CXR, clinical and laboratory findings. The association between detection of pneumococcus and case status was assessed through logistic regression, adjusted for age, sex, antibiotic pre-treatment, HIV and site. Results:

\begin{tabular}{|l|l|l|l|l|l|l|}
\hline & \multicolumn{2}{|l|}{ CXR-AC cases, $\mathrm{N}=526^{*}$} & \multicolumn{2}{l|}{ Controls** } & \multirow{2}{*}{ aOR } & \multirow{2}{*}{ 95\% Cl } \\
\cline { 1 - 4 } & $n$ & $\%$ & $n$ & $\%$ & & \\
\hline NP PCR & 364 & 73.7 & 3093 & 77.1 & 1.70 & $1.16,2.48$ \\
\hline NP culture & 260 & 52.3 & 2884 & 70.0 & 0.92 & $0.66,1.27$ \\
\hline IS PCR & 189 & 70.8 & 168 & 75.7 & 0.91 & $0.56,1.49$ \\
\hline IS culture & 102 & 22.7 & 102 & 33.0 & 0.84 & $0.40,1.75$ \\
\hline
\end{tabular}

$* \mathrm{~N}$ varies by availability of results

**Community controls ( $\mathrm{N}=4244)$ for NP comparison; "non-pneumonia" cases $(\mathrm{N}=333)$ for IS comparison

Among all cases, IS detected an additional 5\% of subjects with Spn, above and beyond what was detected by NPS. Conclusions: The presence of Spn in NPS or IS was not associated with CXR-AC. Serotype-specific analyses may reveal an association between particular Spn types in NPS or IS and CXR-AC.

No conflict of interest

ISPPD-0490

New Pneumococcal Diagnostics---further ahead or more confused?

\section{EVALUATION OF CONFIRMED STREPTOCOCCUS PNEUMONIAE CASES AND CONCORDANCE OF SEROTYPES ACROSS NASOPHARYNGEAL AND INDUCED SPUTUM SPECIMENS IN THE PERCH STUDY}

The PERCH Study Group ${ }^{1}$

${ }^{1}$ International Vaccine Access Center, The Johns Hopkins Bloomberg School of Public Health, Baltimore, USA

Background and Aims: The Pneumonia Etiology Research for Child Health (PERCH) project is a 7-country casecontrol study evaluating the etiology of hospitalized WHO defined-severe or very severe pneumonia among children $28 \mathrm{~d}-59 \mathrm{~m}$ of age. Case specimens include lung aspirates (LA), blood cultures (BCX), nasopharyngeal (NP) swabs, induced sputum (IS), and pleural fluid (PF).

Methods: We assessed concordance of pneumococcal (Spn) serotype cultured from sterile sites (Microbiologically Confirmed Pneumococcal Pneumonia - MCPP) and IS and NP.

Results: Thirty-six MCPP cases had serotyping data from sterile-site pneumococcal isolates (33 BCx, 2 LA, 1 PF). Thirty of 35 MCPP cases with an NP specimen isolated Spn (86\%); serotypes were concordant in 25 (83.3\%) compared to sterile-site isolates. Pneumococcus was identified in 10 of $25(40 \%)$ IS specimens, and $8(80 \%)$ were concordant. Antibiotic pretreatment was higher among subjects contributing IS specimens (64\%) compared to NP (19\%). 


\begin{tabular}{|l|l|l|l|l|l|}
\hline & Sterile site & NP & \multicolumn{2}{l|}{ IS } \\
\cline { 2 - 6 } Serotype & $\mathrm{N}$ & Concordant, N & Discordant, N & Concordant, N & Discordant, N \\
\hline 1 & 4 & 3 & 1 & 1 & 2 \\
\hline 2 & 3 & 3 & 0 & 0 & 0 \\
\hline 9V/9A & 3 & 3 & 0 & 2 & 0 \\
\hline 19A & 4 & 2 & 0 & 1 & 0 \\
\hline 19F & 5 & 4 & 0 & 0 & 0 \\
\hline Other* & 17 & 10 & 4 & 4 & 0 \\
\hline Total & 36 & $\mathbf{2 5}$ & $\mathbf{5}$ & $\mathbf{8}$ & $\mathbf{2}$ \\
\hline
\end{tabular}

$* N=1$ serotypes $13,14,24,39,46,12 F, 16 F, 18 C, 35 B, 7 F, 6 A . N=2$ serotypes $5,6 A / 6 B, 9 V$

Conclusion: Overall NP-sterile site serotype concordance was $71.4 \%$. Poor isolation rates affected overall IS concordance (32\%). A substantial fraction of all MCPP cases will test negative on NP or IS specimens for the disease causing serotype.

No conflict of interest

ISPPD-0158

New Pneumococcal Diagnostics---further ahead or more confused?

\section{USE OF SEROTYPE-SPECIFIC URINE ASSAY TO DETERMINE THE COURSE OF A HOSPITAL OUTBREAK OF STREPTOCOCCUS PNEUMONIAE COMPLICATED BY INFLUENZA A}

C. Sheppard ${ }^{1}$, E. Miller ${ }^{1}$, N. Fry ${ }^{1}$, J. Bendig' ${ }^{2}$, J. Clark², M. Slack ${ }^{1}$, T. Harrison ${ }^{1}$

${ }^{1}$ Respiratory and Vaccine Preventable Bacteria Reference Unit, Public Health England - Colindale, London, United Kingdom; ${ }^{2}$ Department of Microbiology and Virology, Epsom and St Helier University Hospitals NHS Trust, London, United Kingdom

Background and Aims: An outbreak of pneumococcal infection complicated by concomitant Influenza A on an elderly care ward was detected. Six patients with hospital-acquired respiratory infections and two with cultureconfirmed bacteraemia were identified. We sought to determine if patients had the same pneumococcal serotype and infection control measures were successful.

Methods: Isolates were serotyped using standard methods and sent for MIC testing. Urines were tested with BinaxNOW and Bioplex 14-plex serotype-specific pneumococcal antigen assays. Respiratory viral PCR/typing was performed on throat swabs. Amoxicillin prophylaxis was started.

Results: Isolates were serotype 6C and erythromycin resistant.

$\mathrm{ND}=$ not detected, shaded $=$ post prophylaxis

\begin{tabular}{|l|l|l|l|l|l|l|}
\hline Age & $\begin{array}{l}\text { Onset symp- } \\
\text { toms }\end{array}$ & Sample & $\begin{array}{l}\text { Pneumococcal } \\
\text { BinaxNOW }\end{array}$ & $\begin{array}{l}\text { Bioplex } \\
\text { Serotype }\end{array}$ & $\begin{array}{l}\text { Viral swab } \\
\text { Date }\end{array}$ & $\begin{array}{l}\text { Influenza PCR/ } \\
\text { Typing }\end{array}$ \\
\hline 87 & $18 / 05 / 2013$ & urine & Negative & ND & $24 / 05 / 2013$ & Positive A (H3) \\
\hline 82 & $20 / 05 / 2013$ & urine & Positive & 6 A/6C & $24 / 05 / 2013$ & Positive A (H3) \\
\hline 89 & $20 / 05 / 2013$ & urine & Positive & 6 A/6C & $24 / 05 / 2013$ & Positive A (H3) \\
\hline 88 & $22 / 05 / 2013$ & $\begin{array}{l}\text { urine (BC } \\
\mathbf{2 2 / 5 )}\end{array}$ & Positive & 6 A/6C & $24 / 05 / 2013$ & Negative \\
\hline 76 & $22 / 05 / 2013$ & urine & Positive & $\mathbf{6 A / 6 C}$ & $24 / 05 / 2013$ & Negative \\
\hline 93 & $22 / 05 / 2013$ & $\begin{array}{l}\text { urine (BC } \\
\mathbf{2 2 / 5 )}\end{array}$ & Positive & $\mathbf{6 A / 6 C}$ & $24 / 05 / 2013$ & Negative \\
\hline 86 & $23 / 05 / 2013$ & urine & Negative & ND & $24 / 05 / 2013$ & Positive A (H3) \\
\hline 93 & $23 / 05 / 2013$ & urine & Negative & ND & & \\
\hline 91 & $30 / 05 / 2013$ & urine & Negative & ND & $24 / 05 / 2013$ & Positive A (H3) \\
\hline 82 & $30 / 05 / 2013$ & urine & Negative & ND & $30 / 05 / 2013$ & inhibited \\
\hline 95 & $30 / 05 / 2013$ & urine & Equivocal & $\mathbf{1}$ & $30 / 05 / 2013$ & negative \\
\hline
\end{tabular}

Conclusion: This utility of the serotype-specific assay in determining the course of a complicated respiratory outbreak was demonstrated. We determined that infection control measures were effective in containing the pneumococcal spread, i.e. the 6C outbreak had ended, when further co-incidental pneumococcal BinaxNOW positive case appeared.

No conflict of interest 


\title{
DIFFERENTIATION OF STREPTOCOCCUS PNEUMONIAE FROM MITIS GROUP STREPTOCOCCI BY MOLECULAR AND BIOCHEMICAL IDENTIFICATION METHODS
}

\author{
R. Singh ${ }^{1}$, W. Demczuk ${ }^{1}$, A. Griffith ${ }^{1}$, A. McGeer ${ }^{2}$, J. Kellner ${ }^{3}$, G. Zhanel ${ }^{4}$, B. Lefebvre ${ }^{5}$, I. Martin ${ }^{1}$
}

${ }^{1}$ National Microbiology Laboratory, Public Health Agency of Canada, Winnipeg, Canada; ${ }^{2}$ Department of Microbiology, Mount Sinai Hospital, Toronto, Canada; ${ }^{3}$ Department of Pediatrics, Alberta Children's Hospital, Calgary, Canada; ${ }^{4}$ Faculty of Medicine University of Manitoba, Department of Medical Microbiology and Infectious Diseases, Winnipeg, Canada; ${ }^{5}$ Microbiologie, Laboratoire de Santé Publique du Québec, Ste-Annede-Bellevue, Canada

Background and Aims: Traditional biochemical and phenotypic identification methods for Streptococcus pneumoniae such as bile solubility, optochin susceptibility, colony morphology and alpha-hemolysis on blood agar do not consistently differentiate pneumococci from other Mitis group streptococci. This study evaluates biochemical and molecular assays for the identification of Streptococcus pneumoniae from other Mitis group streptococci. Methods: A total of 328 isolates of S. pneumoniae or Streptococcus spp were submitted for identification and serotyping from 2012 to 2013 from respiratory sites $(n=118)$, non-sterile clinical sites $(n=207)$ and sterile sites ( $n$ $=3$ ). Biochemical assays included bile solubility and optochin susceptibility testing; and molecular assays included sequencing of $r p o \mathrm{~B}$ and detection of $c p s \mathrm{~A}$. Serotypes for the S. pneumoniae isolates were determined by Quellung reaction and all isolates were tested with Omnisera (Statens Serum Institute). Sequencing of the tuf genes and detection of the lytA genes were also performed on a sub-set of isolates.

Results: Organisms identified by rpoB sequencing included: S. pneumoniae $(n=216)$; S. pseudopneumoniae $(n=80)$; $S$. mitis $(n=30) ; S$. parasanguinis $(n=1)$; and one isolate was undetermined. Of the $S$. pneumoniae identified, 212 isolates were non-typable by Quelling reaction and 4 were serotyped (serotype $31, n=2$; serotype $7 \mathrm{~F}$, $\mathrm{n}=1$; serotype $23 \mathrm{~F}, n=1$ ). Preliminary data from tuf gene sequencing and detection of the lytA genes supported $r p o B$ sequencing results.

Conclusions: A combination of biochemical and molecular assays are required for the differentiation of $S$. pneumoniae from other Mitis group streptococci.

No conflict of interest

ISPPD-0344

New Pneumococcal Diagnostics---further ahead or more confused?

\section{COMPARISON OF LABORATORY PERFORMANCE AND EFFECT OF CULTURE CONDITIONS ON ISOLATION AND IDENTIFICATION OF STREPTOCOCCUS PNEUMONIAE AMONG LABORATORIES IN INDIA}

S. Amarnath ${ }^{1}$, S. Joshi ${ }^{1}$, T.D. Chugh ${ }^{2}$, K.D. Gandhi ${ }^{3}$, V.A. Indumathi ${ }^{4}$, A. Madhuwanti ${ }^{5}$, R. Mukhopadhyay ${ }^{6}$, S. Muralid-

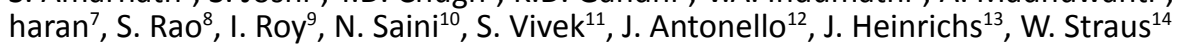

${ }^{1}$ Microbiology, Manipal Cure \& Care, Bangalore, India; ${ }^{2}$ Microbiology, BL Kapoor Hospital, New Delhi, India; ${ }^{3}$ Microbiology, Shanti Mukund Hospital, New Delhi, India; ${ }^{4}$ Microbiology, M S Ramaiah, Bangalore, India; ${ }^{5}$ Microbiology, Metropolis, Pune, India; ${ }^{6}$ Microbiology, GD Institute, Kolkata, India, ${ }^{7}$ Microbiology, St John's Hospital, Bangalore, India; ${ }^{8}$ Microbiology, Microbiology Lab, Mumbai, India; ${ }^{9}$ Microbiology, EEF Hospital, Kolkata, India, ${ }^{10}$ Microbiology, Pushpanjali Hospital, New Delhi, India; ${ }^{11}$ Microbiology, Yashoda Hospital, Bangalore, India, ${ }^{12}$ BARDS, Merck Research Laboratories (MSD), West Point, USA; ${ }^{13}$ Vaccine Basic Research, Merck Research Laboratories (MSD), West Point, USA; ${ }^{14}$ Scientific Affairs, Merck Research Laboratories (MSD), West Point, USA

Background and Aims: The isolation of Streptococcus pneumoniae (Sp) from clinical specimens requires proper growth and identification of colony phenotype, on blood-containing agar plates. In India, where access to sheep blood agar is limited, this poses a challenge. We compared: 1) the ability of ten laboratories in five Indian cities to isolate and identify Sp in complex mixtures provided by a central source; 2 ) sheep blood agar to other commonly used agar types; and 3) the effect of transport on the laboratories ability to isolate the bacteria.

Methods: Laboratories received duplicate swabs containing either pure Sp, or Sp in mixed cultures with a second ("contaminating") bacterium. One swab was immediately shipped back to the central laboratory to assess the impact of shipping on culture viability. The second swab was cultured on sheep-blood, human-blood and one additional agar plate of each laboratories choosing.

Results: 46/60 (77\%) of cultures containing only Sp were correctly identified. In specimens where Sp was present in mixed culture (with a "contaminating" organism), the proportion of isolates in which Sp was correctly identified varied, with most variability attributed to the particular "contaminating organism rather than the media type. There was no discernible impact of temperature (4-6 degrees centigrade) controlled shipping on the isolation of Sp from culture swabs.

Conclusion: These data suggest that Sp may be isolated from agar prepared with human blood, in settings where sheep blood is not available. Furthermore, these results highlight the difficulties inherent in correctly identifying pathogens in mixed cultures in developing world settings.

No conflict of interest 
ISPPD-0410

New Pneumococcal Diagnostics---further ahead or more confused?

\section{HIGH RATES OF STREPTOCOCCUS PNEUMONIAE CARRIAGE IN SALIVA OF ELDERLY DETECTED USING MOLECULAR} METHODS C.L. Krone ${ }^{1}$, J. van Beek ${ }^{2}$, A.L. Wyllie ${ }^{1}$, N.Y. Rots ${ }^{2}$, A. Oja ${ }^{1}$, M.L.J.N. Chu' ${ }^{1}$, J.P. Bruin ${ }^{3}$, D. Bogaert ${ }^{1}$, E.A.M. Sanders ${ }^{1}$,
K. Trzcinski ${ }^{1}$

${ }^{1}$ Pediatric Immunology and Infectious Diseases, Wilhelmina Children's Hospital University Medical Center Utrecht, Utrecht, Netherlands; ${ }^{2}$ Centre for Immunology of Infectious Diseases and Vaccines, National Institute for Public Health and the Environment (RIVM), Bilthoven, Netherlands; ${ }^{3}$ Diagnostic Microbiology, Regional Laboratory of Public Health, Haarlem, Netherlands

Background and Aims: Pneumococcal disease disproportionally affects the very young and the elderly. Unlike in children, pneumococcal colonization in the elderly is rarely detected by conventional culture of nasopharyngeal swabs. Here, we tested nasopharyngeal and saliva samples for pneumococcal carriage in the elderly using molecular methods.

Methods: Trans-nasal nasopharyngeal, trans-oral nasopharyngeal and saliva samples were obtained from 135 persons aged 60-89 during an episode of influenza-like-illness (ILI) and during recovery, 6-8 weeks later. All samples ( $n=272$ per type) were tested for Streptococcus pneumoniae by conventional culture. Following, all visible plate growth (culture-enriched samples) as well as raw saliva were further processed for DNA extraction. Pneumococcal presence was detected by quantitative-PCR (qPCR) targeting two S. pneumoniae-specific genes, IytA and piaA. Results: S. pneumoniae was cultured from $6(2 \%)$ of 272 trans-nasal, $10(4 \%)$ trans-oral and $6(2 \%)$ saliva samples from 14 of 135 elderly. Ten (4\%) culture-enriched trans-nasal, 27 (10\%) culture-enriched trans-oral and 80 (29\%) culture-enriched saliva samples were positive for $S$. pneumoniae by qPCR. The sensitivity of carriage detection was highest from qPCR of culture-enriched saliva samples (80 of $97,82 \%$ ), followed by raw saliva samples (44 or $45 \%$ ) and culture-enriched trans-nasal and trans-oral samples combined (32 or 33\%). In total, 65 of 135 (48\%) individuals were positive for S. pneumoniae at least once; 52 (39\%) during an ILI, 45 (33\%) after recovery and 32 (24\%) at both sampling events.

Conclusions: Using culture-enriched saliva for the molecular detection of S. pneumoniae greatly increases the sensitivity of pneumococcal carriage detection in the elderly.

No conflict of interest 